\title{
Encuesta nacional sobre los conocimientos impartidos en escuelas de medicina de Colombia sobre rehidratación parenteral en niños eutróficos mayores de un año con deshidratación por enfermedad diarreica
}

\author{
Iván Darío Flórez, Esteban Ramos, Carlos Bernal, Olga Juliana Cuéllar, José William Cornejo \\ Grupo de Investigación en Enfermedades del Niño y el Adolescente, Departamento de Pediatría y Puericultura, \\ Universidad de Antioquia, Medellín, Colombia
}

Introducción. Ante la deshidratación grave por diarrea y la contraindicación para rehidratación oral, la rehidratación en niños debe realizarse por vía intravenosa. La Organización Mundial de la Salud (OMS) recomienda la rehidratación rápida.

Objetivos. Describir los métodos de rehidratación intravenosa enseñados en las escuelas de medicina colombianas y confrontarlos con las recomendaciones de la OMS.

Materiales y métodos. Se elaboró una encuesta para docentes de pediatría en escuelas de medicina. Se hicieron preguntas directas y se detallaron casos clínicos de niños deshidratados para ser resueltos. Se preguntó por las indicaciones para la hidratación intravenosa y la forma de hacerla (volumen, solución, concentraciones y velocidad de infusión).

Resultados. Se aplicaron 41 encuestas (82\% de escuelas). Se mencionaron las contraindicaciones inadecuadas para el tratamiento de rehidratación oral en $41 \%$ de ellas. Se recomendó rehidratación intravenosa rápida en $71 \%$, lenta en $29 \%$ y con bolos en $57 \%$. Menos de la mitad de los encuestados recomendaron adecuadamente el volumen por infundir y, el $85 \%$, la concentración de sodio. En $56 \%$ de las escuelas no se usa glucosa en las soluciones y en $65,9 \%$ usan lactato de Ringer. También, se utilizan solución salina normal, dextrosa con electrolitos y solución polielectrolítica.

Conclusiones. Existen ideas erróneas para contraindicar el tratamiento de la rehidratación oral. La tercera parte de las escuelas indican el tratamiento lento a pesar de la superioridad del rápido en la literatura. Falta uniformidad en los esquemas de tratamiento rápido. Es común la hidratación con bolos, sin sustento en la literatura científica. Es necesario actualizar los conceptos sobre hidratación en las escuelas de medicina y proponer una guía nacional para la rehidratación intravenosa.

Palabras clave: fluidoterapia,soluciones para rehidratación, deshidratación, diarrea, Colombia.

Intravenous rehydration for diarrheal dehydration of eutrophic children: survey of protocols provided at Colombian medical schools

Introduction. In all cases of severe dehydration from diarrhea, WHO recommends rapid rehydration. If oral rehydration in children is contraindicated, intravenous rehydration is recommended for immediate administration. However, methods of intravenous rehydration appear to be inadequately addressed in the medical schools of Colombia.

Objective. Current approaches to oral rehydration were summarized, and instructors were informed concerning current WHO recommendations.

Materials and methods. A survey was designed for pediatric instructors in Colombian medical schools. Direct questions about rehydration methods were included as well as presentation of theoretical clinical situations with dehydrated children. The survey also asked for the conditions necessary for intravenous rehydration and method of administration (volume, solution, concentration and speed of infusion).

Results. Forty-one surveys were included ( $82 \%$ of medical schools in Colombia). Inadequate contraindications for oral rehydration therapy were made in $41 \%$. Rapid and slow intravenous rehydration was recommended in $71 \%$ and $29 \%$, respectively; $57 \%$ recommended fluid bolus to rehydrate. Adequate volumes were recommended by less than half of the respondents and adequate sodium concentration was recommended by $85 \%$. In $56 \%$ of medical schools, glucose was not included in solutions and $66 \%$ use Ringer lactate. Normal saline solution, dextrose solution with electrolytes and polyelectrolytes solutions are also used.

Conclusions. Misconceptions are common concerning the contraindications to oral rehydration therapy. One-third of medical schools promote a slow therapy despite the superiority of the rapid therapy. Uniformity for rapid therapy schemes is lacking. Bolus rehydration is commonly advocated 
despite the fact that this method is unsupported by the literature. Concepts about rehydration must be updated in medical schools and a national guide for intravenous rehydration is recommended.

Key words: Fluid therapy, rehydration solutions, dehydration, diarrhea, Colombia.

La enfermedad diarreica es la segunda causa de muerte en niños menores de cinco años, con cerca de dos millones de casos por año en todo el mundo $(1,2)$. La deshidratación, la más frecuente y temida complicación de la enfermedad diarreica, explica gran parte de las muertes y, por esta razón, el pilar del manejo de la diarrea en niños son su prevención y adecuado tratamiento (3-6).

La rehidratación ha evolucionado desde el origen de la rehidratación intravenosa lenta a mediados del siglo pasado, hasta los años 60 y 70 , cuando en los países no desarrollados apareció la rehidratación intravenosa rápida, la cual tomó gran auge y se expandió en la mayoría de estos países por algunos años, hasta el descubrimiento de la rehidratación oral y sus enormes beneficios: impacto en la morbilidad y mortalidad, disminución de la necesidad de líquidos venosos y disminución de los costos hospitalarios (3-12).

En dicho momento, la rehidratación intravenosa quedó relegada a los casos de deshidratación grave o a aquellos con imposibilidad o contraindicación para la rehidratación oral $(3,13,14)$. En esa época se fortaleció el concepto de que lograr la rehidratación en el menor tiempo posible era ideal, dado que la rápida expansión del espacio extracelular permitía mejorar la perfusión renal y gastrointestinal, lo que se traducía en un rápido y efectivo retorno a la alimentación (4). Varias publicaciones posteriores han consolidado aún más la rehidratación oral y la intravenosa rápida como métodos efectivos y seguros para el tratamiento de los niños deshidratados (13-18).

Por lo descrito, muchos autores han desaconsejado la rehidratación intravenosa lenta, teniendo en cuenta que con ella la rehidratación se completa después de 24 horas, lo que puede producir 0 perpetuar la hiponatremia, prolongar la acidosis y, por lo tanto, retardar la reintroducción de la vía oral y aumentar la duración de la estancia hospitalaria $(4,19,20)$. Pero la rehidratación intravenosa lenta

\footnotetext{
Correspondencia:

Iván Darío Flórez, Departamento de Pediatría y Puericultura, Universidad de Antioquia, Calle 67 № 51-27, quinto piso, Medellín, Colombia

Teléfonos: (574) 263-7885 y 263 9856; fax: (574) 2334466

ivoflorez@gmail.com e ivoflorez@hotmail.com

Recibido: 29/06/10; aceptado:02/06/11
}

ha persistido en el tiempo, principalmente en los países desarrollados e, incluso, es recomendada en reconocidos textos de pediatría $(7,21,22)$.

Por otra parte, en muchos países no desarrollados la rehidratación intravenosa rápida continuó siendo la indiscutible opción de manejo de la deshidratación grave, pero con un punto irresoluto: la solución ideal para la reposición. Se ha sugerido que dicha solución debe contener entre 80 y $130 \mathrm{mmol} / \mathrm{L}$ de sodio, cantidades aceptables de potasio, una base y probablemente una cantidad pequeña de glucosa (4). De acuerdo con lo anterior, las soluciones como el cloruro de sodio al $0,9 \%$, podrían no ser adecuadas, mientras que la solución compuesta de lactato sódico (comúnmente llamada lactato de Ringer, en nuestro medio) y otras soluciones polielectrolíticas deberían ser las recomendadas.

A pesar de que se acepta que los niños con deshidratación no grave deben tratarse con rehidratación oral y que en los niños que se requiere rehidratación intravenosa (graves y contraindicaciones para rehidratación oral), ésta debe administrarse mediante el método rápido $(5,13)$, en nuestro medio se utiliza poco la rehidratación oral -lo cual genera con mayor frecuencia el uso de la rehidratación intravenosa- y aún se usa con frecuencia la rehidratación intravenosa lenta, lo que refleja la necesidad de revisar los conocimientos al respecto.

No existe un consenso sobre la forma de tratar la deshidratación por diarrea y, durante muchos años, la Organización Mundial de la Salud (OMS) ha aportado las directrices sobre el tema y sus recomendaciones, si bien se han basado en opiniones de expertos y se encuentran en mora de revisarse, son las más aceptadas en todo el mundo (13). Siendo la guía de la OMS la más aceptada y divulgada, y ante la ausencia de una mejor, consideramos que es la que se debe seguir, especialmente en países no desarrollados, y, por ello, la rehidratación intravenosa rápida debe ser el pilar del manejo ante la imposibilidad o el fracaso de una rehidratación oral o ante un caso de deshidratación grave (13).

Teniendo en cuenta que los conocimientos sobre la rehidratación en casos de diarrea son adquiridos por los médicos durante su formación, el acercamiento al contenido de la cátedra impartida en las escuelas 
de medicina puede reflejar, de alguna forma, las prácticas a las que están siendo sometidos los niños colombianos que la sufren.

No existe a la fecha ningún estudio sobre los métodos de hidratación enseñados en las escuelas de medicina del país. Nos propusimos aplicar una encuesta nacional sobre los conocimientos impartidos en las escuelas de medicina de Colombia sobre los métodos de rehidratación parenteral del niño deshidratado por diarrea, con el fin de describir los esquemas enseñados y las soluciones utilizadas y, además, comparar estos métodos con los recomendados por la OMS.

\section{Materiales y métodos}

Se hizo una búsqueda en las bases de datos disponibles con los nombres de las Instituciones de Educación Superior de Colombia, con programas activos de pregrado de medicina, obteniéndose la más completa información en la página de internet del Sistema Nacional de Información de Educación Superior del Ministerio de Educación de Colombia (http://snies.mineducacion.gov.co/men/).

Con esta información, se construyó una base de datos con cada una de las instituciones, especificando: nombres de la institución, ciudad sede, números telefónicos de contacto, nombre de los jefes del departamento de pediatría o de los coordinadores del área o sección, nombre de los docentes encargados de enseñar la rehidratación parenteral en niños con diarrea, con sus respectivos números telefónicos y correos electrónicos. Los datos se recolectaron mediante llamadas telefónicas a cada una de las instituciones y departamentos 0 coordinaciones de pediatría, según fuera el caso.

Se diseñó una encuesta para indagar sobre algunos tópicos específicos en rehidratación parenteral de los niños con enfermedad diarreica. Se envió la encuesta por vía electrónica al correo que cada docente determinó para ello, luego de obtener su consentimiento verbal para participar en el estudio por medio telefónico. Se solicitó a los docentes que enviaran la encuesta diligenciada al correo electrónico del grupo de Investigación en las Enfermedades del Niño y el Adolescente (Pediaciencias) del Departamento de Pediatría y Puericultura de la Universidad de Antioquia, administrado por un auxiliar de investigación ajeno al estudio, el cual se encargaba de recibir y archivar la información, de tal forma que los investigadores no conocieron el origen de los formatos respondidos.
La encuesta fue dirigida exclusivamente a los docentes responsables de enseñar la cátedra de rehidratación parenteral en niños con enfermedad diarreica. Cuando eran varios los encargados de dicha cátedra, se decidió, con los jefes de los departamentos o coordinadores de pediatría de cada una de las escuelas de medicina, cuál de ellos respondería la encuesta. Se desarrolló una prueba piloto enviando la encuesta a los docentes de las escuelas de medicina de Medellín (cuatro en total), con base en la cual se realizaron algunos ajustes correspondientes, antes de proceder a su ejecución en el resto del país.

Las preguntas de la encuesta eran abiertas y redactadas en forma clara y concisa. La encuesta constó de dos partes. En la primera parte, se hicieron tres preguntas directas dirigidas a conocer la referencia bibliográfica recomendada a los estudiantes sobre el tema, describir las indicaciones recomendadas para instaurar la rehidratación intravenosa, y describir el esquema y las soluciones utilizadas. En la segunda parte, se detallaron cuatro casos clínicos de niños deshidratados en diferentes situaciones, para ser resueltos por el encuestado: deshidratación grave con choque, deshidratado no grave o niño con algún grado de deshidratación, deshidratado grave sin choque y niño con algún grado de deshidratación por hiperémesis. Se establecieron unas definiciones operativas para la tabulación de los datos recolectados. Las variables analizadas fueron las siguientes: referencias bibliográficas, indicaciones para la rehidratación intravenosa, tiempo de rehidratación, volumen de rehidratación, uso de bolos de cristaloides, composición de electrolitos y denominación de las soluciones usadas.

Las referencias bibliográficas correspondieron a las recomendadas a los estudiantes de pregrado para la enseñanza de rehidratación en niños con diarrea. Se agruparon en ocho grupos: capítulos de libro escritos por nefrólogos o pediatras nefrólogos; capítulos de libro escritos por pediatras no nefrólogos; artículos de revisión publicados en revistas, escritos por nefrólogos o pediatras nefrólogos; artículos de revisión publicados en revistas, escritos por pediatras no nefrólogos; guías de la estrategia de Atención Integrada a las Enfermedades Prevalentes de la Infancia (AIEPI) de la Organización Panamericana de la Salud (OPS) y la OMS o sus adaptaciones (AIEPI/ OPS/OMS); guías de manejo de la diarrea y la deshidratación de difusión internacional (diferentes a AlEPI); documentos universitarios elaborados por los docentes, y otras fuentes. 
Las indicaciones de rehidratación intravenosa fueron cada una de las descritas por los docentes encuestados. El tiempo de rehidratación, cuya información se recolectó en número de horas, se categorizó como lento o rápido. En el método lento la duración del esquema era mayor de seis horas. El método rápido se subdividió en dos esquemas: en el primero, que denominamos método rápido con esquema, el tiempo establecido para completar la rehidratación fue de 2, 406 horas; en el segundo, que denominamos método rápido con bolos, los bolos de cristaloides se administraban hasta lograr la rehidratación, sin un tiempo total preestablecido. Igualmente, se analizó la frecuencia de utilización de bolos con cristaloides en estados de deshidratación sin choque. Se evaluó el volumen utilizado en los casos de algún grado de deshidratación y deshidratación grave, midiéndolo en mililitros por kilogramo de peso, y se categorizó mediante los siguientes intervalos: menor de $50 \mathrm{ml} / \mathrm{kg}, 50$ a $99 \mathrm{ml} / \mathrm{kg}, 100$ a $149 \mathrm{ml} /$ $\mathrm{kg}$, mayor de $150 \mathrm{ml} / \mathrm{kg}$, e indeterminado, cuando no quedó claro lo consignado o no hubo forma de calcular el volumen. La composición de sodio, potasio, cloro, glucosa y base de cada una de las soluciones utilizadas, fue analizada y dichos datos se categorizaron en intervalos adecuados y congruentes para cada uno de ellos.

Se analizaron por separado los resultados de las dos partes de la encuesta, y, por ello, los resultados se detallaron con base en las respuestas desglosadas según ambos métodos de preguntas (preguntas directas y resolución de casos clínicos), ya que algunos puntos fueron evaluados tanto en la primera como en la segunda parte de la encuesta.

Un primer análisis de los resultados se restringió a la descripción de lo recomendado por los docentes en las dos modalidades descritas y, el segundo, a la confrontación de los métodos referidos con lo recomendado por la OMS, tanto de las indicaciones para rehidratación intravenosa como de la forma en que se debe realizar ésta; por ello, se examinaron las frecuencias y proporciones de escuelas que utilizan la rehidratación intravenosa rápida de forma similar a la recomendada, así como las que usan otro tipo de rehidratación intravenosa rápida (sin esquema) y las que lo hacían de forma lenta.

La tabulación de los datos fue hecha por dos de los investigadores, quienes en ningún caso conocieron el origen de la encuesta. Cuando hubo discrepancias, se resolvieron en conjunto con los otros investigadores. Como se mencionó, las variables continuas se agruparon en intervalos adecuados para cada una de ellas, y luego se analizaron mediante frecuencias y proporciones. Los datos se analizaron con el paquete estadístico SPSSB, versión 17,0 .

\section{Resultados}

Para el año 2009, se hallaron 56 instituciones de educación superior con programas activos de pregrado de medicina en el Sistema Nacional de Información de la Educación Superior, 19 de carácter oficial $(33,9 \%)$ y 37 de carácter privado $(66,1 \%)$. Del total, nueve tenían programas muy recientes y aún no habian desarrollado actividades en pediatría o sus equivalentes curriculares o estaban inactivos en el sistema, por lo cual no hicieron parte de la muestra. De las 47 encuestas enviadas, $41(87,2$ $\%)$ fueron respondidas y de $6(12,8 \%)$ no se obtuvo respuesta, la mitad de ellas pertenecientes al departamento del Valle del Cauca.

Las referencias bibliográficas sobre rehidratación parenteral en niños con diarrea, recomendadas por los docentes a los estudiantes de pregrado, fueron: capítulos de libro escritos por pediatras no nefrólogos ( $n=34,82,9 \%$ ), guías de AIEPI/OPS/ OMS o adaptaciones de éstas ( $n=24,58,5 \%)$, capítulos de libro escritos por nefrólogos $(n=15$, $36,6 \%$ ), artículos de revista escritos por nefrólogos $(n=9,22 \%)$, guías internacionales (diferentes a AIEPI OPS/OMS) $(n=7,17,1 \%)$, artículos de revista escritos por pediatras no nefrólogos $(n=4$, $9,8 \%$ ), documentos elaborados por los docentes $(n=1,2,4 \%)$ y otras fuentes $(n=3,7,3 \%)$.

Las indicaciones descritas por los docentes para rehidratación intravenosa en las preguntas dirigidas, se presentan en el cuadro 1. En la práctica clínica se indicó la rehidratación intravenosa en las siguientes situaciones: deshidratación grave $(n=38$, $92,7 \%)$, falla de la rehidratación oral $(n=40,97,6$ $\%)$ y algún grado de deshidratación ( $n=2,4,9 \%)$.

En cuanto al método de rehidratación, encontramos que la mayoría de los docentes recomiendan la rehidratación intravenosa rápida con esquema, en la primera parte de la encuesta (con preguntas dirigidas, $n=17,41,5 \%$ y en la segunda parte (resolución de casos clínicos, $n=19,46,3 \%$ ). La rehidratación rápida mediante bolos fue el segundo método recomendado, en las respuestas a las preguntas dirigidas $(n=12,29,3 \%)$ yen las respuestas a los casos clínicos $(n=6,14,6 \%$ ). Seis de los 12 (50 $\%$ ) que respondieron que recomendaban los bolos, respondieron mediante otro esquema ante el caso 
clínico: dos de ellos utilizaban un esquema lento y cuatro de ellos recomendaban un esquema rápido basado en horas.

Por su parte, la rehidratación intravenosa lenta fue recomendada de forma explícita por 12 docentes $(29,3 \%)$ y por $16(39 \%)$ mediante los casos clínicos. Cuatro docentes respondieron que la rehidratación intravenosa rápida era la recomendada, pero en la resolución del caso clínico explicaron un proceso de rehidratación lenta.

Al analizar los resultados según la región del país donde estaba ubicada la escuela, se observó que la recomendación de la rehidratación intravenosa lenta provenía, en todos los casos, de las que se encontraban ubicadas en Bogotá, mientras que en las escuelas del resto de regiones del país recomiendan la rehidratación intravenosa rápida. La rehidratación intravenosa con bolos predominó en las escuelas ubicadas en la Región Caribe: $75 \%$ de las que recomendaron rehidratación intravenosa sin esquema definido, eran de la Costa Atlántica.

Cuadro 1. Indicaciones para el uso de rehidratación intravenosa (respuestas a las preguntas dirigidas)

\begin{tabular}{|c|c|c|}
\hline Indicaciones explícitas & $\begin{array}{l}\text { Número de } \\
\text { facultades }\end{array}$ & Porcentaje \\
\hline Deshidratación grave & 29 & 70,7 \\
\hline $\begin{array}{l}\text { Intolerancia a la vía } \\
\text { oral o vómito incoercible }\end{array}$ & 28 & 68,3 \\
\hline Choque & 21 & 51,2 \\
\hline Falla de la rehidratación oral & 20 & 48,8 \\
\hline Inconciencia o convulsiones & 19 & 46,3 \\
\hline Íleo paralítico & 16 & 39,0 \\
\hline Gasto fecal alto & 15 & 36,6 \\
\hline $\begin{array}{l}\text { Sospecha o confirmación de } \\
\text { abdomen agudo }\end{array}$ & 9 & 22,0 \\
\hline $\begin{array}{l}\text { Rechazo a la vía oral o lesiones } \\
\text { en cavidad oral }\end{array}$ & 8 & 19,5 \\
\hline $\begin{array}{l}\text { Hipopotasiemia sintomática o } \\
\text { distensión abdominal }\end{array}$ & 6 & 14,6 \\
\hline Dificultad respiratoria asociada & 5 & 12,2 \\
\hline Otras & 16 & 39,0 \\
\hline
\end{tabular}

La mayoría recomienda los bolos de cristaloides como parte inicial de los esquemas de rehidratación, bien sea rápida o lenta, tanto en las preguntas dirigidas ( 25 docentes, $53,2 \%$ ), como en los casos clínicos (27 docentes, 57,4\%). El volumen de líquidos para la rehidratación recomendado fue variable, aunque en la mayoría de las ocasiones se encontraba entre 100 y $149 \mathrm{ml} / \mathrm{kg}$ (cuadro 2). Considerando que el volumen ideal de reposición en un niño con algún grado de deshidratación está entre 50 y $100 \mathrm{ml} / \mathrm{kg}$ y para deshidratación grave es entre 100 y $150 \mathrm{ml} / \mathrm{kg}$ (5), 18 (43,9\%) de los encuestados utilizaron un volumen superior al correspondiente a la clasificación del grado de deshidratación (considerando como el volumen ideal entre 50 y $100 \mathrm{ml} / \mathrm{kg}$ ) de acuerdo con las respuestas de las preguntas dirigidas y $13(31,7 \%)$ hicieron lo mismo de acuerdo con las respuestas de los casos clínicos. Por su parte, 8 (19,5\%) y 9 $(22 \%)$ de los docentes en las preguntas dirigidas y en los casos clínicos, respectivamente, utilizaron volúmenes superiores a $150 \mathrm{ml} / \mathrm{kg}$ en caso de deshidratación grave.

La composición de electrolitos de las soluciones más frecuentemente indicadas, tanto en las preguntas dirigidas como en los casos clínicos, contenían concentraciones de sodio entre 101 y 160 $\mathrm{mEq} / \mathrm{L}$, de potasio entre 1 y $10 \mathrm{mEq} / \mathrm{L}$, y de cloro entre 101 y $160 \mathrm{mEq} / \mathrm{L}$, con una base entre 16 y 30 $\mathrm{mEq} / \mathrm{L}$ y sin glucosa. En el cuadro 3 se muestra la composición de las soluciones recomendadas con las respectivas frecuencias y proporciones, y en el cuadro 4 se muestra cada una de las soluciones y su composición. La figura 1 muestra los nombres de las soluciones que más frecuentemente se indicaron, tanto en forma explícita como en los casos clínicos y sus respectivas proporciones. En la mayoría de las escuelas de medicina se prefiere el lactato de Ringer como primera opción para la rehidratación intravenosa, seguido, en su orden, por la solución salina al 0,9\%, mezclas con agua con dextrosa al $5 \%$ con electrolitos, solución

Cuadro 2. Volumen de rehidratación según el grado de deshidratación

\begin{tabular}{|c|c|c|c|c|c|c|c|c|}
\hline \multirow{2}{*}{$\begin{array}{l}\text { Volumen de rehidratación } \\
(\mathrm{ml} / \mathrm{kg})\end{array}$} & \multicolumn{4}{|c|}{ Algún grado de deshidratación } & \multicolumn{4}{|c|}{ Deshidratación grave } \\
\hline & \multicolumn{2}{|c|}{$\begin{array}{c}\text { Preguntas dirigidas } \\
\mathrm{n} \quad(\%)\end{array}$} & \multicolumn{2}{|c|}{ Casos clínicos } & \multicolumn{2}{|c|}{$\begin{array}{c}\text { Preguntas dirigidas } \\
\mathbf{n} \quad(\%)\end{array}$} & \multicolumn{2}{|c|}{$\begin{array}{c}\text { Casos clínicos } \\
\text { n }(\%)\end{array}$} \\
\hline$<50$ & - & & 9 & (22) & - & & 1 & $(2,4)$ \\
\hline $50-99$ & 7 & $(17,1)$ & 9 & (22) & - & & 1 & $(2,4)$ \\
\hline $100-149$ & 18 & $(43,9)$ & 12 & $(29,3)$ & 19 & $(46,3)$ & 23 & $(56,1)$ \\
\hline$\geq 150$ & - & & 1 & $(2,4)$ & 8 & $(19,5)$ & 9 & $(22)$ \\
\hline Indeterminado & 16 & (39) & 10 & $(24,4)$ & 14 & $(34,1)$ & 7 & $(17,1)$ \\
\hline
\end{tabular}


Cuadro 3. Concentraciones de glucosa y electrolitos en las soluciones recomendadas por los docentes en la modalidad de preguntas dirigidas y en la resolución de casos clínicos

\begin{tabular}{|c|c|c|}
\hline Concentración recomendada & $\begin{array}{c}\text { Mediante preguntas } \\
\text { dirigidas } \\
\mathbf{n}(\%)\end{array}$ & $\begin{array}{c}\text { Mediante resolución } \\
\text { casos clínicos } \\
\text { n (\%) }\end{array}$ \\
\hline \multicolumn{3}{|l|}{ Sodio (mEq/L) } \\
\hline$<75$ & $1(2,4)$ & $2(4,9)$ \\
\hline $75-100$ & $12(29,3)$ & $11(26,8$ \\
\hline $101-160$ & $23(56,1)$ & $25(61,0)$ \\
\hline$>160$ & - & - \\
\hline Indeterminado & $5(12,2)$ & $3(7,3)$ \\
\hline \multicolumn{3}{|l|}{ Potasio (mEq/L) } \\
\hline Sin potasio & $3(7,3)$ & $5(12,5)$ \\
\hline $1-10$ & $23(56,1)$ & $25(61,0)$ \\
\hline $11-20$ & $8(19,5)$ & $7(17,1)$ \\
\hline$>20$ & $1(2,4)$ & $1(2,4)$ \\
\hline Indeterminado & $6(14,6)$ & $3(7,3)$ \\
\hline \multicolumn{3}{|l|}{ Glucosa (mmol/L) } \\
\hline Sin glucosa & $23(56,1)$ & $25(61,0)$ \\
\hline $1-75$ & - & - \\
\hline $76-100$ & - & - \\
\hline $101-160$ & $11(26,8)$ & $9(22,0)$ \\
\hline$>160$ & $2(4,9)$ & $4(9,8)$ \\
\hline Indeterminado & $5(12,2)$ & $3(7,3)$ \\
\hline \multicolumn{3}{|l|}{ Cloro (mEq/L) } \\
\hline$<75$ & - & $1(2,4)$ \\
\hline $75-100$ & $13(31,7)$ & $12(29,3)$ \\
\hline $101-160$ & $23(56,1)$ & $25(61)$ \\
\hline$>160$ & - & - \\
\hline Indeterminado & $5(12,2)$ & $3(7,3)$ \\
\hline \multicolumn{3}{|l|}{ Base $(m E q / L)$} \\
\hline Sin base & $7(17,1)$ & $11(26,8)$ \\
\hline $1-15$ & - & - \\
\hline $16-30$ & $29(70,7)$ & $27(65,9)$ \\
\hline$>30$ & - & - \\
\hline Indeterminado & $5(12,2)$ & $3(7,3)$ \\
\hline
\end{tabular}

polielectrolítica y varias mezclas de solución salina al $0,9 \%$ con agua con dextrosa al $5 \%$.

Hubo alguna discrepancia entre la bibliografía recomendada y las respuestas a las preguntas dirigidas en cuanto al método de rehidratación. Para el caso de la bibliografía de la AIEPI/OMS/OPS o adaptaciones, 24 encuestados las recomendaron, pero respondieron luego a favor de la rehidratación intravenosa rápida (que es la recomendada en esta fuente), y en las preguntas dirigidas, sólo 17 de ellos (70\%).

\section{Discusión}

La rehidratación oral partió en dos la historia del tratamiento de la enfermedad diarreica aguda pero, simultáneamente con su aparición, se consolidó la rehidratación intravenosa rápida como una estrategia segura de manejo de los casos en los cuales no era posible utilizar la primera. Precisamente, el mayor logro de la rehidratación intravenosa rápida es que facilitó la reintroducción precoz de la vía entérica en los casos de deshidratación grave para continuar el proceso de rehidratación mediante la rehidratación oral $(19,20)$. Pero, a pesar de esto, en este estudio se encontró que aún se recomiendan esquemas de manejo con rehidratación intravenosa lenta, lo cual se relaciona con mayores tiempos de estancia hospitalaria, mayor demora en el reinicio de la vía oral y mayores costos, entre otras situaciones indeseables $(19,20)$.

Se encontró que la bibliografía recomendada por los docentes es variada debido a la existencia de publicaciones que recomiendan ambos métodos de rehidratación intravenosa. La mayor parte de la bibliografía recomendada se basaba en capítulos de libro escritos por pediatras, o en guías de AIEPI/ OPS/OMS, aunque los capítulos de libro y artículos 
Cuadro 4. Composición de las soluciones formuladas en la encuesta

\begin{tabular}{lcccccc}
\hline Solución & $\begin{array}{c}{\left[\mathrm{Na}^{+}\right]} \\
(\mathbf{m E q} / \mathbf{L})\end{array}$ & $\begin{array}{c}{\left[\mathrm{K}^{+}\right]} \\
(\mathbf{m E q} / \mathbf{L})\end{array}$ & $\begin{array}{c}{[\mathrm{Cl}]} \\
(\mathbf{m E q} / \mathbf{L})\end{array}$ & $\begin{array}{c}{[\mathrm{Base}]} \\
(\mathbf{m m o l} / \mathbf{L})\end{array}$ & $\begin{array}{c}{[\mathrm{Glucosa})} \\
(\mathbf{m m o l} / \mathbf{L})\end{array}$ & $\begin{array}{c}\text { Osmolaridad } \\
(\mathbf{m O s m} / \mathbf{L})\end{array}$ \\
\hline Lactato de Ringer & 130 & 4 & 109 & 28 & - & 271 \\
Solución salina al 0,9\% & 154 & - & 154 & - & - & 308 \\
Solución polielectrolítica & 90 & 20 & 80 & 30 & 111 & 331 \\
Solución 1:1 sin potasio & 77 & - & 77 & - & 139 & 293 \\
Solución 1:1 con potasio & 77 & $10-20$ & $87-97$ & - & 139 & $313-333$ \\
Solución 1:1 con potasio y base & 107 & $10-20$ & $87-97$ & 30 & 139 & $373-393$ \\
Solución 2:1 sin potasio & 102 & - & 102 & - & 92 & 296 \\
Solución 1:2 sin potasio & 51 & - & 51 & - & 185 & 287 \\
Agua con dextrosa al 5\% con electrolitos & $40-80$ & $10-30$ & $50-100$ & - & 278 & $378-488$ \\
\hline
\end{tabular}

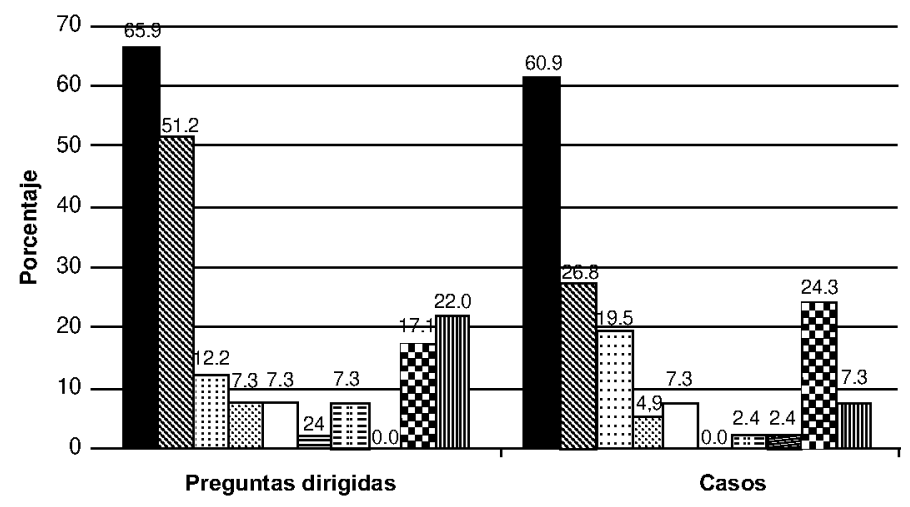

- Lactato de Ringer

Solución salina normal $0.9 \%$

国 Solución polielectrónica 90 (pizarro) $0.9 \%$

B Solución 1:1 sin potasio

$\square$ Solución $1: 1$ con potasio

目 Solución 1:1 con potasio y base

目 Solución 2:1 sin potasio

Solución $1: 2 \sin$ potasio

Agua dextrosada $5 \%$ con electrolitos

III Indeterminada

Figura 1. Soluciones preferidas para rehidratación parenteral.

de revistas escritos por nefrólogos son también insumos importantes para algunas escuelas. Habitualmente, estos últimos son promotores de la rehidratación intravenosa lenta, por lo que se ha sugerido que puede ser inapropiado que estos subespecialistas sean los responsables de estas revisiones $(19,20)$. Por las condiciones de nuestro país, y por el interés de implementar la estrategia AIEPI, se puede pensar que la bibliografía más importante fuera la proveniente de esta estrategia, pero los capítulos de libro superaron en frecuencia a las guías de AIEPI/OPS/OMS, lo cual se explica porque suelen ser revisiones narrativas detalladas y no sólo explican el método de rehidratación, sino, en general, toda la enfermedad. Además, muchas de estas revisiones se basan en bibliografía de AIEPI/OPS/OMS.

Es llamativo que hubo disociación entre la teoría y lo que se cree puede ser la práctica, puesto que $30 \%$ de los docentes que daban como referencia bibliográfica las guías de AIEPI/OPS/ OMS, enseñaban y aplicaban la rehidratación intravenosa lenta recomendada por otros textos de pediatría. Es claro que la causa de ello es la falta de uniformidad en la bibliografía mundial, ya que en la literatura científica proveniente de la OMS recomiendan un método de rehidratación $(5,13)$ y en textos importantes de pediatría se recomienda otro $(21,22)$.

En consonancia con la literatura científica, las indicaciones más frecuentes de la rehidratación intravenosa -0 la contraindicación de la rehidratación oral- fueron: deshidratación grave, falla de la rehidratación oral (por intolerancia a la vía oral o gasto fecal alto), trastornos del estado de conciencia, convulsiones, íleo paralítico, incapacidad para beber y la sospecha o confirmación de un abdomen agudo $(3,5,13,23$ 28). Hallamos otras indicaciones, aunque menos frecuentes, consideradas controvertidas 0 incluso no aceptables, como distensión abdominal, hipopotasiemia, dificultad respiratoria asociada (sin especificar su grado), deshidratación previa durante el episodio actual, sepsis, trastornos electrolíticos, edad menor de tres meses, peso menor de $4,5 \mathrm{~kg}$, pérdida continua de peso o falta de entrenamiento para la rehidratación oral. La enseñanza de contraindicaciones innecesarias para la rehidratación oral en nuestras escuelas puede ser uno de los factores que contribuya a 
la poca divulgación de este tratamiento y debe reevaluarse prioritariamente.

En la práctica clínica, por su parte, la mayoría de los docentes indicó la rehidratación intravenosa ante la deshidratación grave o falla en la rehidratación oral. Algunos docentes intentaban la rehidratación oral cuando había deshidratación grave, lo cual es recomendado por algunos autores (29). En la práctica cotidiana, sin embargo, tal vez la causa más frecuente para indicar la rehidratación intravenosa son las dificultades logísticas para implementar la oral. Se requiere personal adecuadamente adiestrado en suministrar las sales de rehidratación oral y evaluar el éxito de la misma, además de tiempo suficiente para evaluar la progresión durante la observación (24-28). Tal indicación no fue frecuente entre las descritas en la encuesta, precisamente porque no responde a condiciones médicas.

Uno de los puntos que se consideró crítico en este trabajo fue la evaluación de los métodos de rehidratación intravenosa en las diferentes regiones del país. La rehidratación intravenosa rápida ha demostrado ser eficaz y segura en muchos países no desarrollados; por lo tanto, es la recomendada por la OMS para la rehidratación intravenosa de niños con deshidratación grave, con falla o contraindicación de la rehidratación oral $(13,15,16)$. Ésta se desarrolla durante un periodo habitualmente menor de seis horas en niños mayores de un año. A pesar de que algunos métodos de rehidratación rápida incluyen un tiempo de infusión hasta de 12 horas en menores de un año, para efectos de esta encuesta, la rehidratación intravenosa rápida se definió como aquella con una duración menor de seis horas, para no confundirla con la intravenosa lenta y porque todos los casos clínicos que se expusieron se desarrollaban en niños mayores de un año.

Dos terceras partes de las escuelas indican la rehidratación intravenosa rápida (aunque sólo entre 41 y $46 \%$ definen tiempos y volúmenes claros según el grado de deshidratación) y, una tercera parte, la lenta. Esto se relaciona con la falta de recomendación o de inclusión de las guías de OMS/OPS o AIEPI y sus adaptaciones en algunas escuelas del país. Las escuelas de medicina ubicadas en la capital del país recomiendan la rehidratación intravenosa lenta y éstas son las que recomiendan con menor frecuencia la bibliografía relacionada con AIEPI/OPS/OMS. Si bien existen las dos posturas en la literatura científica, cada vez más guías de manejo reconocidas mundialmente, diferentes a las de OMS e incluso divulgadas en países desarrollados, recomiendan la rehidratación intravenosa rápida sobre la lenta $(30,31)$. Llamó la atención la utilización de bolos de rehidratación intravenosa sin esquema definido para rehidratar, recomendación que no tiene sustento en la literatura científica.

En unas pocas encuestas se formularon esquemas de mantenimiento según la fórmula de Holliday-Segar para "corregir" la deshidratación, utilizando soluciones de agua con dextrosa y electrolitos (sodio, cloro y potasio) en cantidad variable. La interpretación de los esquemas de mantenimiento como métodos para la corrección de la deshidratación, ha sido descrita previamente y a ella se le ha adjudicado la persistencia de la deshidratación y el desarrollo de otros trastornos electrolíticos, como hiponatremia grave $(32,33)$.

Más de la mitad de las escuelas indican bolos de cristaloides durante los esquemas de rehidratación, bien sea antes de una rehidratación intravenosa lenta o de una rápida, lo cual es recomendado en algunas publicaciones y guías de manejo $(3,13,15,34)$. Sin embargo, cabe resaltar que aunque deben ser imprescindibles en los casos de los niños que se presentan con choque, los bolos o cargas rápidas, por sí solos, no corrigen todo el déficit hidroelectrolítico y, por lo tanto, luego de su utilización debe instaurarse un esquema para reponer el déficit.

En general, se calcula que el volumen de déficit de agua en los niños con deshidratación por diarrea, se correlaciona con el porcentaje de pérdida de peso corporal y, a su vez, con los signos clínicos de deshidratación $(35,36)$. Se observó que con alguna frecuencia se corrige la deshidratación con volúmenes de soluciones mayores que el déficit calculado. Lo anterior responde a que la mayoría de las veces no se diferenció el volumen según el grado de deshidratación y se indicaron en todos los casos esquemas para deshidratación grave $(100 \mathrm{ml} /$ $\mathrm{kg}$ o más). Aunque estos esquemas no parecen ser perjudiciales en los niños eutróficos y previamente sanos con algún grado de deshidratación, pueden serlo en aquellos con enfermedad renal o cardiaca, o desnutrición aguda grave (37).

Las respuestas obtenidas en las preguntas dirigidas y en los casos clínicos, fueron similares en la mayoría de los casos. No obstante, llamó la atención que la mitad de los encuestados que respondieron que rehidrataban con bolos (sin esquema), al situarse frente a un caso clínico, 
intentaban la rehidratación con un esquema definido, bien fuera lento o rápido. Nos atrevemos a postular que al responder la pregunta dirigida muy posiblemente lo hacian con base en lo que normalmente hacen en la práctica, pero, con el caso clínico específico, trataban de utilizar un esquema definido en la literatura científica que ellos recomendaban. Percibimos un interés de algunos de los docentes por aplicar los esquemas lentos que tienen sustento en tradicionales capítulos de libro, a pesar de que hubiesen respondido de otra forma, lo cual sugiere un respeto importante por estas citas bibliográficas $(21,22)$.

Como se mencionó, se desconoce la solución ideal para la rehidratación intravenosa. Se han probado varias soluciones en los ensayos clínicos para rehidratar niños con diarrea, con diferentes concentraciones de sodio, potasio, cloro, glucosa y bases (lactato, acetato o bicarbonato) $(38,39)$.

Los primeros estudios realizados durante las epidemias de cólera, favorecieron el uso de soluciones polielectrolíticas (con mayor contenido de potasio y con glucosa), comparadas con el lactato de Ringer $(4,40,41)$. Por ello, Hirschorn sugirió que las soluciones para rehidratación intravenosa en diarreas que no fueran por cólera deberían tener un mayor contenido de sodio y potasio que las utilizadas para el mantenimiento, y la glucosa parece más importante de lo que tradicionalmente se ha considerado (4).

La OMS recomienda el lactato de Ringer 0 , en su defecto, la solución de cloruro de sodio al 0,9\% seguida de la rápida introducción de la rehidratación oral $(5,13)$. Igualmente, la World Gastroenterology Organisation (WGO) recomienda el lactato de Ringer para la rehidratación intravenosa rápida (31). En algunos países, como Costa Rica $(42,43)$, Colombia $(44,45)$ y Argentina $(46,47)$, se ha utilizado la solución polielectrolítica (que tiene la misma composición de electrolitos y glucosa que el suero oral estándar con $90 \mathrm{mEq} / \mathrm{L}$ y $20 \mathrm{mEq} / \mathrm{L}$ de potasio), que ha demostrado ser segura y efectiva, ha sido aceptada por la OPS como una opción para la rehidratación intravenosa rápida $(5,48)$ y hace parte de las recomendaciones en guías de algunos países como Argentina (49).

No existen ensayos clínicos controlados que muestren cuál solución es más efectiva y segura. La solución polielectrolítica posee menor cantidad de sodio y mayor cantidad de potasio, así como cuenta con glucosa en su composición y, por ello, evitaría los episodios de hipoglucemia asociados a la deshidratación grave $(4,50)$. Estas características son las que la hacen atractiva para algunos autores en Latinoamérica (40-46). Los posibles defectos de estas soluciones de lactato de Ringer y cloruro de sodio al 0,9\%, en comparación con la solución polielectrolítica, son su baja o nula concentración de potasio y la ausencia de glucosa, que aumenta el riesgo de hipoglucemia (42-49), predominantemente en los lactantes. En esta encuesta se encontró que la mayoría de las soluciones utilizadas se encontraban en los valores adecuados de osmolaridad y de concentraciones de electrolitos, exceptuando la glucosa que fue poco frecuente en las soluciones (39\% de los docentes la incluyeron en sus respuestas).

Las soluciones más utilizadas fueron las dos recomendadas por la OMS. Sin embargo, se debe tener en cuenta que este esquema se recomienda siempre y cuando se reintroduzca rápidamente la rehidratación oral. Por lo anterior, en los casos en los que esto no se pueda garantizar, puede ser preferible la solución polielectrolítica al lactato de Ringer, con el fin de favorecer la rápida reposición del potasio y, por lo tanto, una rápida reintroducción de los alimentos. El mayor defecto de la solución polielectrolítica es que no se encuentra disponible en todo el territorio colombiano y sólo en los departamentos de Antioquia y Santander se puede tener alguna disponibilidad.

Sólo seis escuelas $(12,8 \%)$ no atendieron a la encuesta, por lo cual nos atrevemos a afirmar que los resultados son representativos de los conocimientos impartidos a los estudiantes de medicina del país. Vale la pena resaltar que las preguntas abiertas permitieron que los encuestados describieran los conocimientos que imparten, sin sugerir respuestas y aporta gran validez a los resultados.

Las debilidades de este estudio están dadas por todas aquellas inherentes a las encuestas abiertas, tales como la variabilidad y la incomprensión de algunas respuestas que no fueron claras, Io cual dificultó la tabulación de algunos datos. Es importante, además, recalcar que nos vimos en la obligación de limitar los casos a niños mayores de un año por todo lo descrito en cuanto a la dificultad para definir un tiempo ideal de rehidratación y ello nos limita en la extracción de conclusiones sobre lo recomendado para niños menores, lo cual es una desventaja importante del estudio.

Por otro lado, los resultados de la encuesta no necesariamente son fiel reflejo de la práctica diaria 
en las instituciones de salud. Además, la práctica médica cotidiana está influenciada, no sólo por los conocimientos adquiridos durante la formación, sino también por la literatura médica continuada, la disponibilidad de recursos logísticos y de personal, y la preferencia del personal de salud y de los pacientes, entre otros factores.

Por último, los esquemas que se están enseñando en las escuelas en la actualidad, sólo se materializarán y divulgarán en el transcurso de los próximos dos a cuatro años, cuando los estudiantes se gradúen y ejerzan su profesión, y por ello, es probable que la realidad en la mayoría de los centros donde se atienden nuestros niños sea distinta, ya que los esquemas de hidratación aplicados en la actualidad serán una mezcla de varias generaciones de enseñanzas impartidas en las escuelas médicas en los últimos veinte o treinta años.

Consideramos que encuestar a los docentes de todas nuestras escuelas es el primer paso para evaluarnos, con el fin de corregir conductas innecesarias o inadecuadas y, así, generar intentos de creación de protocolos y guías de manejo específicos para nuestro país o de adaptación local de las guías internacionales si es el caso.

Este estudio permitió concluir que los conocimientos que se imparten en las escuelas de medicina de Colombia respecto a la rehidratación parenteral en niños con diarrea y deshidratación, son muy heterogéneos. A pesar de que hay gran sustento en la literatura científica de la eficacia y seguridad de la rehidratación intravenosa rápida, continúa enseñándose el tratamiento lento en una gran proporción de las escuelas de medicina.

\section{Agradecimientos}

A Luz María Gómez, secretaria de la Sociedad de Pediatría de Antioquia, por facilitar los datos de varios de los profesores y miembros de la Sociedad Colombiana de Pediatría encuestados. A los pediatras y profesores encuestados que se tomaron la molestia de invertir tiempo de sus actividades cotidianas para responder la encuesta de la forma más objetiva posible.

\section{Conflicto de intereses}

Los autores declaramos no tener ningún conflicto de intereses.

\section{Financiación}

El estudio fue financiado con recursos propios del grupo de investigación Pediaciencias y del
Departamento de Pediatría y Puericultura de la Facultad de Medicina de la Universidad de Antioquia.

\section{Referencias}

1. Kosek M, Bern C, Guerrant RL. The global burden of diarrhoeal disease, as estimated from studies published between 1992 and 2000. Bull World Health Organ. 2003;81:197-204

2. Black RE, Morris SS, Brice J. Where and why are 10 million children dying every year? Lancet. 2003;361:2226-34.

3. King CK, Glass R, Bresee JS, Duggan C. Managing acute gastroenteritis among children: Oral rehydration, maintenance, and nutritional therapy. MMWR Recomm Rep. 2003;52:1-16

4. Hirschhorn $\mathbf{N}$. The treatment of acute diarrhea in children: An historical and physiological perspective. Am J Clin Nutr. 1980;33:637-63.

5. Benguigui Y, Bernal C, Figueroa D. Manual de tratamiento de la diarrea en niños. Washington DC: Organización Panamericana de la Salud; 2008. p. 288

6. Victora CG, Bryce J, Fontaine O, Monasch R. Reducing deaths from diarrhoea through oral rehydration therapy. Bull World Health Organ. 2000;78:1246-55.

7. Holliday M. The evolution of therapy for dehydration: Should deficit therapy still be taught? Pediatrics. 1996;98:171-7.

8. Darrow DC, Pratt EL, Flett J, Gamble AH, Wiese HF. Disturbances of water and electrolytes in infantile diarrhea. Pediatrics. 1949;3:129-56

9. Darrow DC. Therapeutic measures promoting recovery from the physiologic disturbances of infantile diarrhea. Pediatrics. 1952;9:519-33.

10. Talbot NB, Crawford JD, Butker AM. Homeostatic limits to safe parenteral fluid therapy. $N$ Eng $J$ Med. 1953;248:1100-8.

11. Rao MC. Oral rehydration therapy: New explanations for an old remedy. Annu Rev Physiol. 2004;66:385-417.

12. Bellemare S, Hartling L, Wiebe N, Russell K, Craig WR, McConnell $\mathrm{D}$, et al. Oral rehydration versus intravenous therapy for treating dehydration due to gastroenteritis in children: A meta-analysis of randomized controlled trials. BMC Med. 2004;2:1-8.

13. World Health Organization. The treatment of diarrhoea: A manual for physicians and other senior health workers. Geneva: World Health Organization; 2005

14. Sperotto G, Carrazza FR, Marcondes E. Treatment of diarrheal dehydration. Am J Clin Nutr. 1977;30:1447-56.

15. Pizarro D. Tratamiento parenteral de la deshidratación en niños con diarrea. Bol Med Hosp Infant Mex. 1986;43:51521.

16. Reid SR, Bonadio WA. Outpatient rapid intravenous rehydration to correct dehydration and resolve vomiting in children with acute gastroenteritis. Ann Emerg Med. 1996;28:318-23

17. Assadi F, Copelovitch L. Simplified treatment strategies to fluid therapy in diarrhea. Pediatr Nephrol. 2003;18:1152-6. 
18. Davidson G, Barnes G, Bass D, Cohen M, Fasano A, Fontaine $\mathrm{O}$, et al. Infectious diarrhea in children: Working Group Report of the First World Congress of Pediatric Gastroenterology, Hepatology, and Nutrition. J Pediatr Gastroenterol Nutr. 2002;35:S143-50.

19. Holliday M. The evolution of therapy for dehydration: Should deficit therapy still be taught? Pediatrics. 1996;98:171-7.

20. Holliday MA, Friedman AL, Wassner SJ. Extracellular fluid restoration in dehydration: A critique of rapid versus slow. Ped Nephrol. 1999;13:292-7.

21. Siegel NJ. Fluids, electrolyte and acid base. In: Rudolph CD, Rudolph A, Hostetter M, Lister G, Siegel N, editors. Rudolph's Pediatrics. 21th edition. Norwalk, CT: AppletonLange; 2004. p. 1644-59.

22. Seigal NJ, Carpenter T, Gaudio KM. Fluid and electrolyte management. In: Crochetti M. Barone M, editors. Oski's essential pediatrics. 2nd edition. Philadelphia: Lippincott Williams and Wilkins; 2004. p. 18-23.

23. Bhutta Z. Acute gastroenteritis in children. In: Kliegman RM, Behrman RE, Jenson HB, Stanton BF, editors. Nelson textbook of pediatrics. 18th edition. Philadelphia, $\mathrm{Pa}$ : Saunders Elsevier; 2007. p 1605-17.

24. Conners GP, Barrer WH, Mushlin Al, Goepp JG. Oral versus intravenous: Rehydration preferences of pediatric emergency medicine fellowship directors. Pediatr Emerg Care. 2000;16: 335-8

25. Snyder JD. Use and misuse of oral therapy for diarrhea Comparison of US practices with American Academy of Pediatrics recommendations. Pediatrics. 1991:87:28-33.

26. Reis EC, Goepp JG, Katz S, Santosham M. Barriers to use of oral rehydration therapy. Pediatrics. 1994;93:708-11.

27. Ozuah PO, Avner JR, Stein RE. Oral rehydration, emergency physicians, and practice parameters: A national survey. Pediatrics. 2002;109:259-61.

28. Sociedad Española de Urgencias en Pediatría. Utilización de la rehidratación oral en urgencias. Encuesta Nacional An Pediatr (Barc). 2003;60:243-8.

29. Murphy MS. Guidelines for managing acute gastroenteritis based on a systematic review of published research. Arch Dis Child. 1998;79:279-84

30. National Institute for Health and Clinical Excellence. Diarrhoea and vomiting caused by gastroenteritis: Diagnosis, assessment and management in children younger than 5 years. Clinical guideline 84. London: NICE, 2009

31. Farthing $\mathbf{M}$, Lindberg $\mathbf{G}$, Dite $\mathbf{P}$, Khalif I, Salazar-Lindo E, Ramakrishna BS, et al. World Gastroenterology Organization practice guideline: Acute diarrea. March 2008. Fecha de consulta: 24 de mayo de 2010. Disponible en http://www. worldgastroenterology.org/assets/downloads/ en/pdf/guidelines/01_acute_diarrhea.pdf

32. Liebelt EL. Clinical and laboratory evaluation and management of children with vomiting, diarrhea and dehydration. Curr Opin Pediatr. 1998;10:461-9

33. Friedman AL, Ray PE. Maintenance fluid therapy: What it is and what it is not. Pediatr Nephrol. 2008;23:677-80

34. Brewster DR. Dehydration in acute gastroenteritis. J Paediatr Child Health. 2002;38:219-22.
35. Gorelick MH, Shaw KN, Murphy KO. Validity and reliability of clinical signs in the diagnosis of dehydration in children. Pediatrics. 1997;99:e6.

36. Steiner MJ, DeWalt DA, Byerley JS. Is this child dehydrated? JAMA. 2004;291:2746-54

37. Kerpel-Fronius E. Volume and composition of the body fluid compartments in severe infantile malnutrition. J Pediatr. 1960;56:826-33

38. Jucá CA, Rey LC, Martins CV. Comparison between normal saline and a polyelectrolyte solution for fluid resuscitation in severely dehydrated infants with acute diarrhoea. Ann Trop Paediatr. 2005;25:253-60.

39. Levy JA, Bachur RG. Intravenous dextrose during outpatient rehydration in pediatric gastroenteritis. Acad Emerg Med. 2007;14:324-31.

40. Mujibur-Arman M, Majad MA, Monsur KA. Evaluation of two intravenous rehydration solutions in cholera and noncholera diarrhoea. Bull World Health Organ. 1979;57:97781.

41. Gutman RA, Drutz DJ, Whalen GE, Watten RH. Double bind fluid therapy evaluation in pediatric cholera. Pediatrics. 1969;44:922-31.

42. Pizarro D, Posada G, Mohs E. Rehidratación rápida por la vía endovenosa en niños deshidratados por diarrea. Bol Med Hosp Infant Mex. 1980;37:315-74

43. Posada G, Pizarro D. Rehidratación por vía la endovenosa rápida con una solución similar a la recomendada por la OMS para rehidratación oral. Bol Med Hosp Infant Mex. 1986;43:463-9.

44. Bernal C, Valencia ML, Bastidas M, Gómez C. Hidratación parenteral rápida en pacientes deshidratados por enfermedad diarreica aguda. latreia. 1989;2:207-13.

45. Bernal C, Correa AC, García G. Hidratación parenteral con solución 90 (solución Pizarro) en niños deshidratados por enfermedad diarreica. latreia. 1994;7:118-25.

46. Ferrero FC, Osorio F, Voyer LE, González H, Macario MF, Cabeza M. Rehidratación endovenosa rápida con 90 $\mathrm{mmol} / \mathrm{L}$ de sodio en niños deshidratados por diarrea. Bol Med Hosp Infant Mex. 1991;48:474-8

47. Escobal N, Rodríguez J, Figueroa C, Fraquelli L, Marciano B, Abelanz M, et al. Balance hidroelectrolítico en hidratación rápida en lactantes con diarrea aguda. Bol Med Hosp Infant Mex. 1995;52:231-8.

48. Bello O, Sehabiague G, Prego J, Caligaris M, Vivas S, Stagno F. Análisis prospectivo de la norma actual de rehidratación intravenosa: ¿la solución 90 es la solución? Rev Soc Bol Ped. 1997:36:S2-S10.

49. Afazani A, Beltramino D, Bruno ME, Cairoli H, Caro $\mathrm{MB}$, Cervetto JL, et al. Diarrea aguda en la infancia. Actualización sobre criterios de diagnóstico y tratamiento. Consenso Nacional Argentino. Documento Sociedad Argentina de Pediatría. Fecha de consulta: 9 de abril 2010. Disponible en: http://www.sap.org.ar/docs/profesionales/ consensos/diarreagu.pdf

50. Hirschhorn N, Lindenbaum J, Greenough WB, Mahmood Alam S. Hypoglycemia in children with acute diarrhoea. Lancet. 1966;2:128-33. 